\author{
${ }^{1,2}$ E.K. Baygazieva, ${ }^{1,3}$ N.N. Yesmurzayeva, \\ ${ }^{1,3}$ G.S. Tatykhanova, ${ }^{2}$ G.A. Mun, ${ }^{4}$ V.V. Khutoryanskiy, \\ ${ }^{1,3}$ S.E Kudaibergenov* \\ ${ }^{1}$ Laboratory of Engineering Profile, K.I. Satpayev Kazakh National Technical University, Almaty, Kazakhstan \\ ${ }^{2}$ al-Farabi Kazakh National University, Almaty, Kazakhstan \\ ${ }^{3}$ Institute of Polymer Materials and Technology, Almaty, Kazakhstan \\ ${ }^{4}$ Reading School of Pharmacy, University of Reading, Reading, UK \\ *E-mail: skudai@mail.ru

\section{Polymer Protected Gold Nanoparticles: Synthesis, Characterization and Application in Catalysis}

\begin{abstract}
This review discusses the stabilization of gold nanoparticles (AuNPs) by nonionic, anionic, cationic and amphoteric polymers. The protocols used for synthesis of AuNPs in aqueous and organic solvents are described. Size, shape and morphology of AuNPs are characterized by various physicochemical methods. Application aspects of polymer-protected AuNPs in catalysis are outlined.

Keywords: gold nanoparticles, hydrophilic polymers, stabilization, catalysis
\end{abstract}

\section{Introduction}

In recent years metal nanoparticles have attracted a significant interest due to their large surface-tovolume ratios and quantum size effects [1]. Nanostructured metal colloids have been prepared by mechanical grinding of bulk metals, e.g. "top-down" methods [2]. However this method has a difficulty to obtain uniformly distributed particle size. The "bottom-up" methods (or wet chemical preparation of nanoparticles) rely on the chemical reduction of metal salts, electrochemical pathways, etc. Nowadays a large variety of stabilizers are known, such as donor ligands, polymers, different surfactants that are used to control the growth of the nanoparticles and to prevent them from aggregation [3].

Most of polymers used for protecting of AuNPs are of nonionic, anionic and cationic nature [4-7]. The mechanism of stabilization of nanoparticles by polymers involves two basic modes. One of them is electrostatic stabilization based on the double layer repulsion between the particles. The other mode is the steric stabilization of colloidal particles achieved by physical adsorption of macromolecules on the surface of particles.

This paper presents a review of literature on preparation and characterization of AuNPs stabilized by various water-soluble polymers. Some ap- plication aspects of polymer-protected AuNPs in catalysis are also discussed.

\section{AuNPs stabilized by nonionic hydrophilic polymers \\ Poly(N-vinylpyrrolidone) (PVP) is one of the} frequently used nonionic polymers for stabilizing metal nanoparticles. A simple synthesis of AuNPs from a sonication assisted thermal dissociation reaction of a gold salt $\mathrm{HAuCl} 4 \cdot 3 \mathrm{H} 2 \mathrm{O}$ in the presence of PVP in water is described [8]. The size of AuNPs, ranging from 10 to $110 \mathrm{~nm}$, is easily controlled by varying the content of PVP in solution (0.01-10 $\mathrm{g} \cdot \mathrm{dL}-1)$ relative to an aqueous salt precursor.

Synthesis of gold and silver hydrosols was carried out in one-step process by reduction of aqueous solutions of metal salts using PVP [9]. Both kinds of metal nanoparticles were obtained without addition of any other reducing agent, at low temperatures and using water as the reaction medium. Shape, size, and optical properties of the particles were tuned by changing the employed $\mathrm{PVP} /$ metal salt ratio.

The AuNPs and AgNPs were stabilized by PVP having different molecular weights in both aqueous and organic solvents $[10,11]$. The absorption spectra, size, and morphology of AuNPs and AgNPs were determined. The catalytic activity of AuNPs 
and $\mathrm{AgNPs}$ deposited on $\mathrm{Al} 2 \mathrm{O} 3$ by impregnation method was evaluated with respect to hydrogen peroxide decomposition [12]. PVP-protected Pt-core bimetallic $\mathrm{Pt} / \mathrm{Au}$-shell $(\mathrm{Pt} / \mathrm{Au})$ nanoparticles were prepared using multi-step reduction of $\mathrm{HAuCl} 4$ and $\mathrm{H} 2 \mathrm{PtCl} 6$ alternately by hydrogen adsorbed on platinum atoms [13].

Authors [14] have reported a novel route to produce AuNPs/polyvinyl alcohol (PVA) hydrogels in which AuNPs functionalized with carboxylic groups act as crosslinkers through esterification reaction with the PVA hydroxyl groups. To avoid nanoparticles precipitation during this process, the reaction mixtures were "frozen" in a well-dispersed particle state resulting in the physical gelation of PVA through the application of one freezing-thawing cycle.

Three amino acid derivatives of PVA modified by Gly, Ala and Val were obtained through a onestep esterification reaction from the corresponding isobutyryl chloride [15]. These thermoresponsive PVA derivatives were good dispersing agents for AuNPs in water. The AuNP was covered with a thick organic shell formed by thermoresponsive PVA derivatives. The composites of PVA derivatives and AuNPs showed better salt-resistance than those stabilized by neat PVAs.

Well-dispersed gold colloids stabilized by polyacrylamide (PAAm) were obtained by reduction of gold salt with PAAm [16]. The structure and composition of these nanoparticles were characterized by X-ray photoelectron spectroscopy (XPS), TEM, FTIR, and UV-Vis absorption spectra.

Nanocomposite materials consisting of colloidal AuNPs were embedded in thermo-responsive poly(N-isopropylacrylamide) (PNIPAm) hydrogels [17]. Thiol groups that bind to both $\mathrm{Au} 3+$ ions and colloidal Au are incorporated into the side-chains of the PNIPAm hydrogels through copolymerization. Compared with the non-AuNPs containing PNIPAm hydrogels, the AuNPs-PNIPAm nanocomposite hydrogels have shown higher degrees of equilibrium swelling and different temperature-triggered phase transitions.

AuNPs stabilized with PNIPAM were prepared by two distinct routes: (i) conventional free-radical polymerization leading to polymer without any reactive end-group, and (ii) RAFT polymerization with 2-dodecylsulfanylthiocarbonylsulfanyl-2-methyl propionic acid (DMP) as a RAFT agent [18]. PNIPAM with low polydispersity was end-capped with $\alpha$-carboxylic acid and $\omega$-trithiocarbonate that was converted into $\omega$-thiol upon hydrolysis. This hetero-telechelic polymer was effective in stabilizing AuNPs ( 1-5 nm).

Poly(ethylene glycol) (PEG) modified AuNPs were synthesized using a one-solution synchrotron X-ray irradiation method [19]. The impact of process parameters such as PEG molecular weight, PEG/gold molar ratio and X-ray absorbed dose on the structure and morphology of AuNPs were investigated. The size of PEG-modified AuNPs was found to decrease with increasing PEG content in the reaction mixture and X-ray dose. With the capability to monitor the absorption spectra in situ during the fast synthesis process, this approach opens the way to control particle size and distribution accurately. PEG chains with an intermediate length (MW 6000) were found to be optimal for size control and colloidal stability in biologically relevant media. PEG-AuNPs synthesized using X-ray radiation could find interesting applications in nanoparticle-enhanced X-ray tumor imaging and therapy.

TEM micrographs show that the PEG-AuNPs are spherical with $6.1 \pm 1.9 \mathrm{~nm}$ diameter and reasonably narrow size distribution. The Au (111) plane with a planar spacing of $2.32 \AA$ confirms the crystal structure of the nanoparticles. The crystal nature of PEG-AuNPs particles was also confirmed by XRD measurements. The estimated average nanoparticle size from the broadening of the reflection peak (111) was $7.6 \mathrm{~nm}$. The hydrodynamic size measured by DLS for re-dispersed PEG-AuNPs in DI water was $27.9 \pm 8.1 \mathrm{~nm}$. The thickness of the PEG coating was $25 \mathrm{~nm}$. FTIR spectrum of PEG-AuNPs shows that the broad band in the $3200-3600 \mathrm{~cm}^{-1}$ regions is attributed to the $\mathrm{OH}$ group and suggests that hydroxyl groups play a role in linking the nanoparticles to PEG chains. Vibrational bands that are characteristic for $\mathrm{CH} 2$ groups at $2884 \mathrm{~cm}^{-1}$ and the $\mathrm{C}-\mathrm{O}-\mathrm{C}$ stretching band at $1114 \mathrm{~cm}^{-1}$ confirm that PEG chains are immobilized at the nanoparticle surfaces. A low concentration of unmodified PEG macromolecules was very effective in controlling particle size and in stabilizing the AuNPs. Both the PEG chain length and the PEG/Au ratio affected the size, morphology and colloidal stability of the particles.

PEG-coated gold nanoparticles (PEG-AuNPs) were synthesized using a one-step route with rapid microwave heating [20]. Homogeneous nucleation of the primary gold particles is enhanced by increasing the applied microwave power during the initial 
stage of the synthesis, increasing the temperature ramping rate $\left(R_{r}\right)$ and resulting in decreased size and improved uniformity of the synthesized PEGAuNPs. The UV-Vis absorption spectra of PEGAuNPs synthesized with the two different $R_{r}$ values and size distributions of PEG-AuNPs synthesized at $R_{r}=124.2^{\circ} \mathrm{C} / \mathrm{min}$ reveal that as the $R_{r}$ value increases from $34.2^{\circ} \mathrm{C} / \mathrm{min}$ to $124.2^{\circ} \mathrm{C} / \mathrm{min}$, the Full Width Half Maximum (FWHM) of the absorbance profile narrows and the maximum peak ( $\lambda \max )$ value shows a blue shift from $528 \mathrm{~nm}$ to $521 \mathrm{~nm}$, indicating the formation of colloidal PEG-AuNPs with a smaller average size and narrower size distribution. The TEM images and the corresponding size distribution of PEG-AuNPs are in a good agreement with the UV-Vis results. These results indicate that uniform PEG-AuNPs with an average diameter of $14.3 \pm 2.5 \mathrm{~nm}$ are produced at $R_{r} \approx 124.2^{\circ} \mathrm{C} / \mathrm{min}$. UVVis absorbance spectra of PEG-AuNPs in the presence of $1.0 \mathrm{M} \mathrm{NaCl}$ show that no significant shifts are observed within at least $24 \mathrm{~h}$, indicating that the resulting PEG-AuNPs are stable under these conditions.

Authors [21] synthesized AuNPs capped with iodine and PEG to provide effective enhancement for X-ray computed tomography (CT) imaging. The methoxy PEG-iodine-capped AuNPs were prepared through the chemisorption of iodine and substitution of methoxy PEG-SH onto the surface of gold nanoparticles, and severe aggregation in TEM was not observed. The binding energies of $\mathrm{Au} 4 \mathrm{f} 7 / 2$ and I $3 \mathrm{~d} 5 / 2$ of the methoxy PEG-iodine-capped AuNPs were recorded as $84.1 \mathrm{eV}$ and $619.3 \mathrm{eV}$, respectively. The binding energy shift of methoxy PEG-iodine-capped AuNPs would be resulted from the chemisorption between gold nanoparticles and iodine atoms. The methoxy PEG-iodine-capped AuNPs have higher enhancement compared to PEG-capped gold nanoparicles in the same amount of gold in vitro. After injection of methoxy PEG-iodine-capped AuNPs into mice, a dramatic contrast enhancement in the heart, aorta, liver, and kidney was observed; this was maintained for up to 5 days, and there was no evidence of apparent toxicity. Methoxy PEGiodine-capped AuNPs might be a good candidate as a CT contrast agent for blood pool imaging, and this will also contribute to the prolongation of a circulation time in the blood for X-ray CT imaging.

A series of thiol-PEG-modified AuNPs (PEGylated AuNPs) with different PEG molecular weights $(\mathrm{Mw})$ were synthesized [22]. The saturated capping density, charge-screening ability, and stability of the PEGylated AuNPs were then examined. The results showed that high-Mw PEG stabilized the AuNPs and screened the surface charge better than low-Mw PEG, but the latter showed higher saturated capping density. More importantly, PEG exhibited the maximum coagulation concentration (MCC) and critical stabilization concentration (CSC) in the stabilizing process of the AuNPs. Thiol-PEG acted as an AuNPs stabilizer only when its concentration was higher than the CSC. Otherwise, thiol-PEG accelerated AuNPs aggregation, which reached the peak level at the MCC. These results were significant in recognizing the influence of thiol-PEG on the stability of AuNPs.

The effect of PEG surface density on the aggregation behavior of AuNPs in water, dichloromethane (DCM) and the model biological media, phosphate-buffered saline (PBS) and PBS with bovine serum albumin (BSA) was evaluated [23]. PEG-coated AuNPs were stable at salt concentrations of 0.15-1 M, whereas citrate capped AuNPs aggregated immediately under these conditions. In the case of re-dispersion in DCM and $\mathrm{H} 2 \mathrm{O}$, there is an approximately linear relationship between the amount of PEG attachment and stability. Lower levels of stability are observed in highly PEG-functionalized AuNPs after re-dispersion in PBS and PBS/BSA compared to DCM and $\mathrm{H} 2 \mathrm{O}$ due to the effect of $\mathrm{NaCl}$.

Competitive adsorption kinetics between thiolated polyethylene glycol (SH-PEG) and mercaptopropionic acid (MPA) on AuNPs were studied using a prototype physical characterization approach that combines dynamic light scattering (DLS) and electrospray differential mobility analysis (ES-DMA) [24].The change in hydrodynamic particle size were measured by DLS in both two component (AuNPs + MPA or AuNPs + SH-PEG) and three component (AuNPs +MPA + SH-PEG) systems. ES-DMA was employed to quantify the surface coverage of SHPEG and establish a correlation between surface coverage and the change in particle size measured by DLS. A change in equilibrium binding constant for SH-PEG on AuNPs at various concentrations of SH-PEG and MPA showed that the presence of MPA reduced the binding affinity of SH-PEG to the AuNPs surface.

Authors [25] reported a fabrication method for making mesoporous colloidal composites in which AuNPs are adsorbed uniformly onto the 
porous polymer microspheres. Poly(ethylene glycol dimethacrylate-co-acrylonitrile) microspheres were used as a mesoporous polymer support system. Particular interest was focused on the effect of their surface area and surface functionality on the anchoring of AuNPs and the morphology of final microspheres.

\section{Stabilization of AuNPs by anionic polymers}

Authors [26] reported the synthesis of poly(acrylic acid) (PAA)-capped gold nanoparticles. The polyelectrolyte-wrapped gold nanoparticles were fully characterized and studied via a combination of techniques, i.e. UV-Vis and IR spectroscopy, dark field optical microscopy, SEM, DLS and zeta potential measurements.

A new methodology is described [27] for the one-step aqueous preparation of highly monodisperse gold nanoparticles with diameters below $5 \mathrm{~nm}$ using thioether- and thiol-functionalized PAA and poly(methacrylic acid) (PMAA). It was shown that PAA and PMAA were the most effective stabilizing polymers and that relatively low molar mass ligands (approximately $2500 \mathrm{~g} / \mathrm{mol}$ ) gave rise to the narrowest particle size distributions.

Hydrophobically modified polyelectrolytes, in particular poly(acrylic acid) modified by butylamine, was used for the controlled single-step synthesis and stabilization of gold nanoparticles [28]. The size and shape of the particles obtained in the presence of unmodified or modified polyelectrolytes were compared. The presence of hydrophobic microdomains at the gold particle surface leads to a better stabilization of gold nanoparticles at higher temperature.

Poly(divinylbenzene-co-acrylic acid) (poly (DVB-co-AA)) hollow microspheres with gold nanoparticles on the interior surfaces were prepared from the gold nanoparticles-coated poly(methacrylic acid) (PMAA@Au@poly(DVB-co-AA))core-shell microspheres by removal of the PMAA core in water [29]. Au nanoparticles-coated PMAA microspheres were formed using in-situ reduction of gold trichloride with PMAA microsphere as a stabilizer via the interaction between carboxylic acid groups and $\mathrm{Au}$ nanoparticles. Gold nanoparticles-coated (PMAA@Au@poly(DVB-co-AA)) microspheres were formed during the distillation precipitation copolymerization of divinylbenzene and acrylic acid in acetonitrile with Au-coated PMAA microspheres as seeds. The PMAA microspheres, Au nanopar- ticles-coated PMAA microspheres, core-shell microspheres, and hollow microspheres with AuNP on the interior surfaces were studied by TEM and SEM. The stabilization to L-cysteine and some catalytic activity of the Au nanoparticles on the inner surface of hollow poly(DVB-co-AA) microspheres were investigated.

The pH-responsive control of the colloidal dispersions of imidazolium cations-modified gold nanoparticles by using PAA has been reported [30]. The gold nanoparticles were precipitated when PAA $(\mathrm{Mw}=25000)$ was added in aqueous solution at $\mathrm{pH}=2.4$. The precipitate was re-dissolved when the solution's $\mathrm{pH}$ dropped below $\mathrm{pH} 1.9$ and rose above $\mathrm{pH}$ 5.5. According to UV-Vis spectra, TEM observations, and DLS analysis, it is indicated that the gold nanoparticles at lower $\mathrm{pH}(\mathrm{pH}<1.9)$ were well re-dispersed in aqueous solution and aggregates with a diameter of $115 \pm 21 \mathrm{~nm}$ of the gold nanoparticles were formed at $\mathrm{pH}>5.5$. The effect of molecular weights of PAA was also studied.

Authors [31] described a simple approach for controllable synthesis of one-dimensional (1D) AuNP assemblies in solution. In the presence of divalent metallic ions, poly(acrylic acid)-1-dodecanethiol-stabilized AuNPs (PAA-DDT@AuNPs)were found to form 1D assemblies in aqueous solution using an ion-templated chelation process. The assemblies are very stable and remain suspended in solution for more than one month without significant aggregation.

The development of thermoresponsive hybrid poly(acrylamide-acrylic acid) microgels within of which the AuNPs were encapsulated has been reported in [32]. The effect of the nanoparticles on the thermosensitivity, morphology, and optical properties of the microgel was evaluated.

Gold nanoparticles of $5 \mathrm{~nm}$ diameter, stabilized with 4-(dimethylamino) pyridine were coated with poly(sodium 4-styrene sulfonate) (PSS) via electrostatic self-assembly [33]. The suspension stability, monitored by the gold surface plasmon band, was studied by varying the $\mathrm{pH}$, the PSS chain length, and the polymer concentration.

\section{Protecting of AuNPs by cationic polymers}

The most of cationic polymers such as poly(ethyleneimine) (PEI) [34], PAAm [35], poly(N,N-diethylaminoethyl methacrylate], (PDEAMA), [36,37] poly(N,N-dimethyl-N,N-diallylammonium chloride) (PDMDAAC), [38] poly(4- 
vinylpyridine) (P4VPy) [39], and other amine-containing polyelectrolytes $[40,41]$ are able to mediate and stabilize gold colloids from a chloroauric acid precursor.

Poly(N-isopropylacrylamide)-b-[poly(ethylacrylate)-g-poly(2-(diethylamino)ethyl meth-acrylate)] (PNIPAM-b-(PEA-g-PDEAEMA)) double hydrophilic graft copolymers were employed to prepare stable colloidal gold nanoparticles in situ with controllable size in aqueous media without any external reducing agent [37]. PDEAEMA side chains served as a reducing agent and stabilizer and PNIPAM segment acted as a hydrated layer to enhance the stability of gold nanoparticles. These gold nanoparticles showed a remarkable colloidal stability without any observable flocculation or aggregation for at least 2 months. The size of gold nanoparticles can be tuned by adjusting the length of PDEAEMA side chains and the molar ratio of $[$ DEAEMA $] /\left[\mathrm{AuCl}_{4}^{-}\right]$. Both the increasing of the length of PDEAEMA side chains and the decreasing of $[\mathrm{DEAEMA}] /\left[\mathrm{AuCl}_{4}^{-}\right]$molar ratio resulted in the reduction of particle of size.

A series of organo-soluble spherical AuNPs were prepared through the reduction of $\mathrm{HAuCl}_{4}$ by $\mathrm{NaBH} 4$ in the presence of amphiphilic polymers that had a hydrophilic hyperbranched polyethylenimine core and a hydrophobic shell formed by many palmitamide (C16) chains [42].

It was found that higher feed ratio of polymer to $\mathrm{HAuCl}_{4}$ and utilization of polymers with higher $\mathrm{C} 16$ density resulted in smaller AuNPs with relatively lower polydispersity. These organo-soluble AuNPs were used as efficient catalysts for the biphasic catalytic reduction of 4-nitrophenol by $\mathrm{NaBH}_{4}$.

Hydrophobically-modified polycations (i.e., $\mathrm{N}$-acylated chitosan) were used to stabilize gold nanoparticles [43]. TEM observations revealed that the modified chitosan gold nanoparticles were well dispersed and spherical in shape with an average size around 10-12 nm in triply-distilled water at $\mathrm{pH}$ 7.4 , whereas the native chitosan gold nanoparticles appeared as clusters with $9.9 \mathrm{~nm}$ as an average diameter and were dispersed only in dilute $\mathrm{HCl}$.

Reverse micelles of star-block copolymers poly(styrene)-block-poly(4-vinylpyridine) (PSb-P4VPy) were used as nanoreactors for preparation of AuNPs [39]. The $\mathrm{HAuCl}_{4} \cdot 3 \mathrm{H}_{2} \mathrm{O}$ embedded within P4VPy micelle core surrounded by PS shell was reduced by anhydrous hydrazine. As a result the AuNPs of small quantum size (ca. $2 \mathrm{~nm}$ ) soluble in toluene were produced.

\section{Amphoteric polymers as stabilizers of AuNPs}

Information on stabilization of AuNPs by amphoteric polyelectrolytes is very limited. A new regular polyampholyte, namely poly-(N,N-diallyl$\mathrm{N}, \mathrm{N}$-dimethylammonium-alt-N-octyl-maleamic carboxylate) is proved to be an efficient reducing and stabilizing agent for the formation of gold colloids [44]. The process of nanoparticle formation was investigated in the absence of any other reducing agent, in water as well as in the microemulsion template phase. In both cases, nanoscale gold particles can be synthesized, while the adsorption of the polymer on the particle surface prevents their aggregation due to electrostatic stabilization.

Water-soluble and durable $\mathrm{Au}$ nanoclusters, smaller than $4 \mathrm{~nm}$ with a narrow size distribution, were supported on a $\mathrm{pH}$ - and solvent-responsive water-soluble polyampholyte (SPES) [45]. The $\mathrm{Au} @$ SPES hybrids displayed clear pH- and solvent-sensitive properties, and exhibited precipitation behavior in response to $\mathrm{pH}$ and solvent changes in aqueous solution.

Furthermore, the recycled catalyst could be redissolved in water for reuse via simple procedures based on the $\mathrm{pH}$-sensitivity of the polyampholyte. By employing these catalysts, aerobic oxidation of alcohols can be carried out in a homogeneous manner and may thus give rise to similar catalytic activities and selectivities as the homogeneous parent system. The phase separation and sensitivity of the SPES-stabilizing Au nanoclusters permitted a facile separation of the clusters from the reaction mixture without any negative aggregation.

Several diblock polyampholytes poly (methacrylic acid)-block-poly(N,N-dimethylaminoethyl methacrylate), PMAA-b-PDMAEMA having different molecular weight and block ratio were used for stabilization of AuNPs [46]. The fabrication of gold nanoparticles was realized in DMF and in THF. The type of solvent has an impact on the particle size of the gold nanoparticles.

The average size of these AuNPs is around $5.1 \pm 2.7 \mathrm{~nm}$ in THF and $3.0 \pm 2.6 \mathrm{~nm}$ in DMF. The size of gold particles is greater in THF because the aggregation of the PDMAEMA blocks is greatly enhanced in low polarity THF compared to DMF.

We have synthesized a series of AuNPs stabilized by various polyampholytes. As seen from Table 1 the average sizes of AuNPs are varied from 11 to $44 \mathrm{~nm}$ while the plasmon resonance spectra of AuNPs are appeared at 530-550 nm. 
Table 1 - The sizes and the absorbance profile of polyampholyte-protected AuNPs

\begin{tabular}{|l|c|c|}
\hline \multicolumn{1}{|c|}{ Polyampholytes } & $\begin{array}{c}\text { The average } \\
\text { size, nm }\end{array}$ & $\begin{array}{c}\text { The maximum } \\
\text { peak value, } \lambda_{\max }\end{array}$ \\
\hline Poly(N,N-dimethylaminoethyl methacrylate-methacrylic acid) & 17 & 540 \\
\hline Poly(vinylbenzyldimethylammonium acetate) & 20 & 550 \\
\hline Poly(vinylbenzyldiethylammonium acetate) & $4-44$ & 550 \\
\hline Poly(N,N-dimethyl-N,N-diallylammonium-alt-N-phenylmaleamic acid & 22 & 530 \\
\hline Poly(N,N-dimethyl-N,N-diallylammonium-alt-N-4-butylphenylmaleamic acid & 11 & 540 \\
\hline
\end{tabular}

\section{Polymer-protected AuNPs in catalysis}

The AuNPs exhibit the catalytic activity due to their large surface area per unit volume or weight of metal than the bulk Au. Haruta and co-authors [47] achieved excellent results in catalysis of CO oxidation with gold nanoparticles deposited on different support. Authors [48] investigated the dependence of the chemical reactivity of AuNPs on the cluster size and shape using a combination of simulation techniques. It was revealed that more atoms of gold situated on the top of the cluster for clusters of smaller size.

There are many methods of catalysts preparation using adsorption, impregnation, precipitation, co-precipitation, deposition-precipitation etc. In particular, co-precipitation and deposition-precipitation methods were used for preparation of gold nanoparticles deposited onto some support $[49,50]$.

Polymer-stabilized metal nanoparticles can act as the most effective catalysts. If the interaction between the macromolecules and metal nanoparticles is strong, there remains no vacant site on the surface of the metal nanoparticles. This means that, when substrate molecules attack the surface of metal particles, they cannot reach the surface of metal because of the absence of a vacant site, i.e., no catalytic reaction occurs on the surface of metal nanoparticles. If the interaction between the macromolecules and metal nanoparticles is weak, then the substrate molecules can replace a coordinating site of the polymer molecule and attack the surface of metal, which results in the prompt catalytic reaction.

As described previously the polymers surrounding the metal nanoparticle play an important role in stabilization and control of their structure. The polymers or stabilizers play an important role to improve the catalytic activity and selectivity of metal nanoparticles, which is similar to the case of protein surrounding the active site of metal enzymes [51]. The polymers usually have interactions with substrate molecules, which can increase the rate of reaction with the substrates and/or increase the selectivity by choosing the particular substrate.

Authors [52] prepared catalyst based on polymer-protected AuNPs by colloidal deposition on the $\mathrm{ZnO}$ supports. PVA stabilized gold nanoparticles deposited on different supports were used for BTX (benzene, toluene and p-xylene) oxidation. The conversion of BTX mediated with $\mathrm{Au} / \mathrm{ZnO}$ is higher than $\mathrm{Au} / \mathrm{Al} 2 \mathrm{O} 3$ and $\mathrm{Au} / \mathrm{MgO}$.

AuNPs stabilized with PVP deposited onto $\mathrm{A} 12 \mathrm{O} 3$ was examined with respect to hydrogen peroxide decomposition under mild conditions [11].

Citrate-reduced AuNPs (average diameter is 14 $\mathrm{nm}$ ) protected by thermoresponsive hyperbranched polyethylenimine with isobutyramide groups (HPEI-IBAm) was used as recyclable responsive catalysts for the reduction reaction of 4-nitrophenol by NaBH4 [53]. By choosing the right capping HPEI-IBAm polymers, the reaction was faster than that catalyzed by AuNPs without capping polymers. Moreover, the thermoresponsive AuNP catalysts were recovered by increasing the temperature above their LCSTs and recycled at least six times with more than $95 \%$ conversion.

A novel nanocomposite was prepared by deposition of carbonate-stabilized $\mathrm{Au}$ nanoparticles (AuNPs) onto the surface of poly(diallyldimethyl ammonium chloride) (PDDA)-coated carboxylated nanocrystalline cellulose (NCC). The hybrid material contained 1.45 wt $\%$ of AuNPs with an average diameter of $2.95 \pm 0.06 \mathrm{~nm}$. The catalytic activity of AuNP/PDDA/NCC for reducing 4-nitrophenol to 4-aminophenol was compared to other Au-supported composites. Activation energy of 9.2 $\mathrm{kJ} \cdot \mathrm{mol}^{-1}$ was recorded for this reaction. The reaction rate constant $\mathrm{k}$ of $(5.1 \pm 0.2) \cdot 10-3 \mathrm{~s}^{-1}$ was comparable to the benchmark literature value obtained using AuNPs ( $<5 \mathrm{~nm}$ in diameter) decorated on a network of crystalline cellulose fibers [54]. 
Authors [55] synthesized AuNPs using highly branched PEI and acylated polyethylenimine (PEIC12) and evaluated their catalytic activity. The AuNPs, in a PEI polyelectrolyte matrix, were found to be astable and active catalyst for the selective reduction of nitro compounds using $\mathrm{NaBH} 4$ under mild reaction conditions. This catalytic system is stable for several months without any obvious colloidal aggregation.

The ability of the AuNPs stabilized by chitosan as a catalyst for the reduction of eosin dye in the presence of $\mathrm{NaBH} 4$ was monitored by means of spectrofluorometry and spectrophotometry [56]. It was found that the $\mathrm{NaBH} 4$-induced reduction of eosin is enhanced in the presence of AuNPs even without a catalyst. Time-resolved fluorescence decay studies also confirmed the reduction of eosin in the presence of AuNPs.

\section{Conclusion}

Hydrophilic polymers have attracted increasing interest as stabilizers for AuNPs since they exhibit many advantages, such as enhancing the long-term stability, tuning the solubility and amphiphilicity, promoting the compatibility and processability, and giving AuNPs special properties. The low inherent toxicity, multifunctionality, high surface area, photophysical and optical properties of AuNPs impart unique attributes that have a great importance in catalysis, chemotherapy, cancer diagnosis and drug delivery. Polymer protected gold nanoparticles can be synthesized by "one step" methods in the presence of nonionic, anionic, cationic and amphoteric polymers. In most cases the functional polymers act as both reducing and stabilizing agents for the gold nanoparticles. The gold particles can be formed in core-shell structure, hydrophobic environment, microemulsions, microspheres and be soluble in both water and organic media. The size, shape and morphology of AuNPs depend on the molecular mass of polymers, polymer/gold molar ratio, solvent nature, $\mathrm{pH}$, temperature etc. The literature survey shows that the lesser considered subject is polyampholyteAuNPs systems. Polymer protected AuNPs are used to mediate decomposition, hydrogenation and oxidation reactions of various substrates.

\section{Acknowledgement}

Financial support from JSC "National Scientific Technological Holding "Parasat" (Agreement No. 529) is greatly acknowledged.

\section{References}

1 Shmidt G. Clusters and Colloids. From theory to applications. Wienheim: VCH; 1994.

2 Gaffet E., Tachikart M., Kedim O. El, Rahouadj R. Nanostructural Materials Formation by Mechanical Alloying: Morphologic Analysis Based on Transmission and Scanning Electron Microscopic Observations //Materials Characterization -1996. - V.36. -P.185-190.

3 Zhou J., Ralston J., Sedev R., Beattie D.A. Functionalized gold nanoparticles: Synthesis, structure and colloidal stability //Journal of Colloid and Interface Science. -2009. - V.331. -P. 251-262

4 Bekturov E.A., Kudaibergenov S.E., Iskakov R.M., Zharmagambetova A.K., Ibraeva Zh.E., Shmakov S. Polymer-protected nanoparticles of metals. Almaty. -2010.

5 Ibrayeva Zh.E., Kudaibergenov S.E., Bekturov E.A. Stabilization of metal nanoparticles by hydrophilic polymers (in Russian). LAP Lambert Academic Publishing, Germany. - 2013. 376 p;

6 Shan J., Tenhu H. Recent advances in polymer protected gold nanoparticles: Synthesis, properties and applications (Review)// Chem. Commun. - 2007. - V. 44. - P.4580-4598.

7 Bajpai S.K., Murali Y.M., Bajpai M. Synthesis of polymer stabilized gold and silver particles //Journal of Nanoscience and Nanotechnology. 2007. - V.7. - P. 2994-3010.

8 Ram S., Agrawal L., Mishra A., Roy S.K. Synthesis and optical properties of surface stabilized gold nanoparticles with poly(vinyl pyrrolidone) polymer molecules of a nanofluid // Advanced Science Letters - 2011. - V. 4(11-12). - P. 3431-3438

9 Hoppe C. E., Lazzari M., Pardinas-Blanco I., Lopez-Quintela M. A. One-Step Synthesis of Gold and Silver Hydrosols Using Poly(N-vinyl-2-pyrrolidone) as a Reducing Agent // Langmuir. - 2006. - P. 7027-7034.

10 Kudaibergenov S. E., Tatykhanova G.S., Baigaziyeva E. Preparation, Characterization and Catalytic Activity of Gold Nanoparticles Stabilized by Hydrophilic Polymers. Proceedings of the International Conference "Nanomaterials: Applications and Properties", NAP-2012, Alushta, Crimea, Ukraine, September 16-22, 2012. Vol.1, No.1, 01PCN-42(P3).

11 Kudaibergenov S.E., Baigaziyeva E.K., Yesmurzayeva N.N., Nurakhmetova Zh.A., Selenova B.S. Synthesis, Characterization and Catalytic Property of Polymer Protected Gold and Silver Nanopar- 
ticles // Proceedings of the international conference nanomaterials: applications and properties. -2013. - V. 2 (1). - P.5.

12 Yesmurzayeva N., Selenova B., Kudaibergenov S. Preparation and catalytic activity of gold nanoparticles stabilized by poly $(\mathrm{N}$-vinylpyrrolidone) and deposited onto aluminum oxide // J. American Nanomaterials. - 2013. - V.1. - P.1-4.

13 Dai J., Yao P., Hua N., Yang P. and Du Y. Preparation and characterization of polymer-protected Pt@Pt/Au core-shell nanoparticles //Journal of Dispersion Science and Technology. - 2007. - V. 28(6). -P. 872-875.

14 Moreno M., Hernandez R., Lopez D. Crosslinking of poly(vinyl alcohol) using functionalized gold nanoparticles// European Polymer Journal. 2010. -V.46. - P. 2099 - 2104.

15 Rui-Cong Wang, Hua-Ji Liu, Jin-Ge Tong, $\mathrm{Yu}$ Chen. Thermoresponsive poly(vinyl alcohol) derivatives: preparation, characterization and their capability of dispersing gold nanoparticles // Polym. Chem. - 2014.

16 Bai J., Li Y., Du J., Wang S., Zheng J., Yang Q., Chen X. One-pot synthesis of polyacrylamide-gold nanocomposite // Materials Chemistry and Physics. -2007. - V.106. - P. 412-415

17 Wang C., Flynn N.T., Langer R. Morphologically Well-defined Gold Nanoparticles Embedded in Thermo-Responsive Hydrogel Matrices // Mat. Res. Soc. Symp. Proc.- 2004. - V. 820. -P. 333-338.

18 Aqil A., Qiu H., Greisch J.F., Jerome R., De Pauw E., Jerome C. Coating of gold nanoparticles by thermosensitive poly(N-isopropylacrylamide) end-capped by biotin //Polymer. - 2008. -V.49. - P.1145-1153.

19 Wang C.H., Liu C.J., Wang C.L., Hua T.E., Obliosca J.M., Lee K.H., Hwu Y., Yang C.H., Liu R.S., Lin H.M., Je J.H., Margaritondo G.. Optimizing the size and surface properties of polyethylene glycol (PEG)-gold nanoparticles by intense X-ray irradiation // J. Phys. D: Appl. Phys. - 2008. -V. 41. -P. 1-8.

20 Seol S.K., Kim D., Jung S., Chang W.S., and Kim J.T. One-Step Synthesis of PEG-Coated Gold Nanoparticles by Rapid Microwave Heating // Journal of Nanomaterials. - 2013. - P. 6.

21 Kim S.H., Kim E.M., Lee C.M., Kim D.W., Lim S.T., Sohn M.H., Jeong H.J.. Synthesis of PEG-Iodine-Capped Gold Nanoparticles and Their
Contrast Enhancement in In Vitro and In Vivo for X-Ray/CT // Journal of Nanomaterials. - 2012. P. 9.

22 Wang W, Wei Q.Q, Wang J, Wang B.C, Zhang S.H, Yuan Z. Role of thiol-containing polyethylene glycol (thiol-PEG) in the modification process of gold nanoparticles (AuNPs): stabilizer or coagulant? // J Colloid Interface Sci. - 2013. - V. 404. - P. 223-229.

23 Manson J., Kumar D., Meenan B.J. and Dixon D. Polyethylene glycol functionalized gold nanoparticles: the influence of capping density on stability in various media. Gold Bull. - 2011. DOI 10.1007/s13404-011-0015-8.

24 Tsai D.H., DelRio F.W., MacCuspie R.I., Cho T.J., Zachariah M.R., Hackley V.A.. Competitive Adsorption of Thiolated Polyethylene Glycol and Mercaptopropionic acid on Gold Nanoparticles Measured by Physical Characterization Methods // Langmuir. - 2010. -V. 26(12). - P.10325-10333

25 Kim Y.J., Kim J.W., Lee J.E., Ryu J.H., Kim J., Chang I.S., Suh K.D. Synthesis and adsorption properties of gold nanoparticles within pores of surface-functional porous polymer microspheres // Journal of polymer science: part a: polymer chemistry. - 2004. - V. 42. - P.5627-5635.

26 Jans H., Jans K., Lagae L., Borghs G., Maes G., Huo Q., Jans H., Jans K., Lagae L., Borghs G., Maes G. and Huo Q. Poly(acrylic acid)-stabilized colloidal gold nanoparticles: synthesis and properties // Nanotechnology. - 2010. - V.21. - P. 455702

27 Wang Z, Tan B, Hussain I, Schaeffer N, Wyatt M.F, Brust M, Cooper A.I. Design of polymeric stabilizers for size-controlled synthesis of monodisperse gold nanoparticles in water // Langmuir. 2007. - V.16. - P.885-895.

28 Note C., Koetz J., Kosmella S., Tiersch B. Hydrophobically modified polyelectrolytes used as reducing and stabilizing agent for the formation of gold nanoparticles // Colloid and Polymer Science. - 2005. - V. 283. - P. 1334-1342.

29 Liu W., Yang X.L., He X.G., Synthesis of poly(divinylbenzene-co-acrylic acid) hollow microspheres with gold nanoparticles on the interior surface // Chinese J. Polym. Sci. - 2009. - V. 27. - P. 275.

30 Naka K., Tanaka H., Chujo Y. pH Responsive Aggregation of Imidazolium Cations-Modified Gold Nanoparticles with Poly(acrylic acid) in Aqueous Solution// Polymer Journal. - 2007. - V. 39. - P. 1122-1127. 
31 Zhu L., Xue D., Wang Z. Metallic Cation Induced One-Dimensional Assembly of Poly(acrylic acid)-1-Dodecanethiol-Stabilized Gold Nanoparticles // Langmuir. - 2008. -V.24 (20). - P. 1138511389.

32 Echeverria C., Mijangos C. Effect of Gold Nanoparticles on the Thermosensitivity, Morphology, and Optical Properties of Poly(acrylamideacrylic acid) Microgels //Macromol. Rapid Commun. - 2009. - P. 1-5.

33 Dorris A., Rucareanu S., Reven L., Barrett C.J., Bruce L. R. Preparation and characterization of polyelectrolyte-coated gold nanoparticles// Langmuir. - 2008. -V. 24(6). - P. 2532-2538.

34 Sun X.P., Zhang Z.L., Zhang B.L., Dong X.D., Dong S.J., Wang E.K. Preparation of Gold Nanoparticles Protected with Polyelectrolyte // Chinese Chemical Letters. - 2003. - V. 14(8). - P. 866 -869 .

35 Sardar R., Bjorge N.S., Shumaker-Parry J.S. pH-controlled assemblies of polymeric aminestabilized gold nanoparticles // Macromolecules. -2008. -V. 41(12). - P. 4347-4352.

36 Chen H., Lentz D.M., Hedden R.C. Solution templating of $\mathrm{Au}$ and $\mathrm{Ag}$ nanoparticles by linear poly[2-(diethylamino)ethyl methacrylate] // J. Nanoparticle Research. - 2012. - V. 14. - P. 690.

37 Feng C., Gu L., Yang D., Hu J., Lu G., Huang X. Size-controllable gold nanoparticles stabilized by PDEAEMA-based double hydrophilic graft copolymer // Polymer. - 2009. -V. 50. - P. 3990-3996.

38 Chen H., Wang Y., Wang Y., Dong S., Wang E. One-step preparation and characterization of PDDA-protected gold nanoparticles // Polymer. 2006. - V. 47. - P.763-766.

39 Li J., Shi L., An Y., Li Y., Chen X., Dong H. Reverse micelles of star-block copolymer as nanoreactors for preparation of gold nanoparticles // Polymer. - 2006. - V.47. - P. 8480-8487.

40 Lebedeva O. V., Kim B.S., Gröhn F., Vinogradova O. I. Dendrimer-encapsulated gold nanoparticles as building blocks for multilayer microshells //Polymer. - 2007. - V. 48. - P.5024-5029.

41 Sun X., Dong S., Wang E. One-step synthesis and characterization of polyelectrolyte-protected gold nanoparticles through a thermal process// Polymer. - 2004. -V. 45. - P.2181-2184.

$42 \mathrm{Hu}$, N., Yin, J.Y., Tang, Q., Chen, Y. Comparative study of amphiphilic hyperbranched and linear polymer stabilized organo-soluble gold nanoparticles as efficient recyclable catalysts in the biphasic reduction of 4-nitrophenol // Journal of Polymer Science. - 2011. - V. 49(17). - P. 38263834.

43 Remant Bahadur KC, Aryal S, Bhattarai SR, Bhattarai N, Kim CH, Kim HY. Stabilization of gold nanoparticles by hydrophobically-modified polycations // J Biomater Sci Polym Ed. - 2006. V.17. - P.579-890.

44 Note C., Koetz J., Wattebled L., Laschewsky A. Effect of a new hydrophobically modified polyampholyte on the formation of inverse microemulsions and the preparation of gold nanoparticles // J. Colloid and Interface Sci. - 2007. - V. 308. - P. 162-169.

45 Li S., Wu Y., Wang J., Zhang Q., Kou Y., Zhang S. Double-responsive polyampholyte as a nanoparticle stabilizer: Application to reversible dispersion of gold nanoparticles //J. Mater. Chem. -2010. - V. 20. - P. 4379-4384.

46 Mahltig B., Cheval N., Gohy J.F., Fahmi A. Preparation of gold nanoparticles under presence of the diblock polyampholyte PMAA-b-PDMAEMA// J. Polym. Res. - 2010. - V. 17. - P. 579-588.

47 Haruta M., Kobayashi T., Sano H., Yamada N. Novel Gold Catalysts for the Oxidation of Carbon Monoxide at a Temperature far Below $0{ }^{\circ} \mathrm{C} / /$ Chemistry Letters. - 1987. -V.16. - P.405-408.

48 Brodersen S.H., Gronbjerg U., Hvolbak B., Schiotz J. Understanding the catalytic activity of gold nanoparticles through multi-scale simulations.// Journal of catalysis. - 2011. - V. 284. P.34-41.

49 Haruta M., Yamada N., Kobayashi T., Iijima S. Gold catalysts prepared by co-precipitation method for low-temperature oxidation of hydrogen and of carbon monoxide // Journal of catalysis -1989. -V.115. - P. 301-309.

50 Tsubota S., Cunningham D., Bando Y., Haruta $\mathrm{M}$. Preparation of nanometer gold strongly interacted with $\mathrm{TiO} 2$ and the structure sensitivity in low-temperature oxidation of $\mathrm{CO} / /$ Preparation of catalysts VI, Scientific bases for the preparation of heterogeneous catalysts. Elsevier Science. - 1995. - V. 227-235.

51 Toshima N. Nanostructured metal clusters in polymeric field as a model of artificial enzyme// Supramolecular Sci. -1998. - V.5. - P. 395-398. 
52 Wang L., Zhang J., Shen Z., Zhao J. Catalytic oxidation of benzene, toluene and p-xylene over colloidal gold supported on zinc oxide catalyst// Catalysis Communication. - 2011. - V.12. P. 859-865.

53 Liu X.-Y., Cheng F., Liu Y., Liu H.J., Chen Y. Preparation and characterization of novel thermoresponsive gold nanoparticles and their responsive catalysis properties // J. Mater. Chem. - 2010. - V.20. - P.360-368.

54 Lam E., Hrapovic S., Majid E., Chong J.H., Luong J.H.T. Catalysis using gold nanopar- ticles decorated on nanocrystalline cellulose // Nanoscale. - 2012. -V. 4(3). - P. 997-1002.

55 Veerakumar P. et al. Polyelectrolyte encapsulated gold nanoparticles as efficient active catalyst for reduction of nitrocompounds by kinetic method// Appl. Catalysis A: General - 2012. - V.439440. - P.197-205.

56 Komalam A., Muraleegharan L.G., Subburaj S., Suseela S., Babu A., George S. Designed plasmonic nanocatalysts for the reduction of eosin $\mathrm{Y}$ : absorption and fluorescence study// International Nano Letters. -2012. - V. 2. - P. 26. 